\title{
Arribada y explotación de la tortuga golfina en la Playa Escobilla, Oaxaca, México
}

\section{Nesting and exploitation of the Olive Ridley Sea Turtle at Escobilla Beach, Oaxaca, Mexico}

\author{
Pedro Cervantes-Hernández ${ }^{1 *}$, Eduardo Pérez-Vives ${ }^{2}$ y Mario Alejandro Gómez-Ponce ${ }^{3}$
}

\begin{abstract}
RESUMEN
Desde 1959, en el Pacífico mexicano, la explotación de Lepidochelys olivacea no se ha evaluado y en la Playa Escobilla, la temporada de arribada está parcialmente documentada. Los temas mencionados fueron analizados con los métodos recomendados en la bibliografía (análisis de la mortalidad y series de tiempo) y para ello, se usó una base de datos de captura total de enero del año 1978 a mayo del año 1990. Se encontraron tendencias netas descendentes en la abundancia de machos y hembras, concluyendo que esta población fue sobreexplotada en el período en estudio. La mortalidad natural fue muy baja en comparación con la mortalidad por pesca, y esta última afectó a ambos sexos con la misma intensidad. Una temporada completa de arribada se delimitó de agosto a enero, con el máximo de abundancia en noviembre. Con base en la bibliografía se encontró que en el Golfo de Tehuantepec (GT), la temporada de arribada transcurrió al mismo tiempo que la temporada reproductiva del camarón café Farfantepenaeus californiensis (de julio a febrero, con máximo desove entre octubre y noviembre). En el GT la temporada de pesca del recurso camarón es de septiembre/octubre a marzo/abril, abarcando completamente la temporada de arribada. La acción conjunta de las pesquerías de tortugas marinas (aquí explicada) y del recurso camarón provocó la sobreexplotación de L. olivacea. La pesquería del recurso camarón hizo de L. olivacea una especie común en la fauna de acompañamiento; por esta razón, antes y en el presente, los excluidores continúan utilizándose.
\end{abstract}

Palabras claves: Escobilla, explotación, estación de arribada, tortugas marinas, Pacífico mexicano.

\begin{abstract}
The exploitation of Lepidochelys olivacea along the Mexican Pacific has not been assessed since 1959. In addition, nesting season at Escobilla beach has only been partially documented. These two issues were analyzed with the methods recommended in the literature (mortality analysis and time series) using a database with total catch from January 1978 to May 1990. Net trends in the abundance of males and females descended over the study period, which led to the conclusion that the population was overexploited. Natural mortality rate was much lower compared to fishery mortality rate, the latter affecting both sexes with the same intensity. A complete nesting

\footnotetext{
Universidad del Mar, Instituto de Recursos. Carretera a Zipolite, Ciudad Universitaria s/n, Distrito de San Pedro Pochutla A. P. 47, C.P. 70902, Puerto Ángel, Oaxaca, México.pch@angel.umar.mx*

Egresado Licenciatura en Biología Marina, Universidad del Mar, Puerto Ángel, Oaxaca, México. México. Km. 9.5 Carretera Carmen Puerto Real. CP. 24157, Ciudad del Carmen, Campeche, México.
}

Unidad de Servicio el Carmen, Instituto de Ciencias del Mar y Limnología, Universidad Nacional Autónoma de
\end{abstract}

Recibido: 7 de setiembre de 2016

Corregido: 19 de mayo de 2017

Aceptado: 22 de mayo de 2017

DOI: http://dx.doi.org/10.15359/revmar.9-1.6 
season spanned from August to January, with maximum abundance in November. As indicated in the literature, the nesting season of the Olive Ridley Sea Turtle in the Gulf of Tehuantepec (GT) coincided with the reproductive season of brow shrimp Farfantepenaeus californiensis (from July to February, with maximum spawning between October/November). In the GT shrimp fishing season is from September/October to March/April, completely overlapping the nesting season. Simultaneous fishing of the marine turtle (explained here) and shrimp caused overexploitation of L. olivacea. Shrimp fishery made L. olivacea a common species in the accompanying fauna. Consequently, turtle excluders were and continue to be used.

Keywords: Escobilla, exploitation, nesting season, marine turtles, Mexican Pacific.

\section{INTRODUCCIÓN}

En 1959, en el Pacífico mexicano, la pesquería de tortugas marinas comenzó con un promedio anual de captura de 500 t, duplicándose en 1960 y alcanzando el máximo en 1968 con 14330 t (Márquez-M. et al. 1982; Trinidad \& Wilson, 2000). El Gobierno Federal mexicano, a través de los Anuarios Estadísticos de Pesca 19801982, mantuvo registros detallados para esta actividad a nivel nacional, estatal, e incluso, en localidades estratégicas como Playa Mazunte (Early-Capistrán, 2014), Puerto Ángel y Puerto Escondido, Oaxaca, México (Fig. 1) (PESCA, 1980-1989). Pero después, en los Anuarios Estadísticos de Pesca 1983-1988, los registros solo se emitieron a nivel estatal (Fig. 1) y en los siguientes tomos desde 1989 hasta la fecha, no se incluyó registro alguno para las tortugas marinas (Fig. 1).

Para las tortugas marinas del Pacífico mexicano, la implementación de vedas inició en 1972 (Márquez-M. et al. 1982). En el Anuario Estadístico de Pesca 1995, se anunció y ratificó la veda permanente a partir del 16 de marzo de 1994 (PESCA, 1995-2007); y preliminarmente, se informó para todas las embarcaciones camaroneras del Océano Pacífico, acerca del uso obligatorio de dispositivos excluidores para tortugas marinas en las redes de arrastre (con entrada en vigor el 19 de marzo de 1996). Las modificaciones a esto último están documentadas en las Normas Oficiales Mexicanas NOM-EM-001-1996 y NOMEM-002-1996 (SEMARNAP, 19961996). Para los excluidores de tortugas marinas, las especificaciones técnicas están contenidas en la NOM-061PESC-2006 (con fecha de publicación del 22 de enero del 2007 y entrada en vigor el 23 de marzo del 2007) (SAGARPA, 2007).

Para México y el estado de Oaxaca, se evidenció una tendencia descendente en los niveles de captura para las tortugas marinas (Fig. 1), que hasta la fecha, nunca ha sido analizada ni explicada a fondo. Para el Pacífico mexicano, algunas evidencias apuntan a que desde 1938, las tortugas marinas han constituido una fracción sustancial en la captura incidental de otras especies comerciales (Seminoff et al. 2008); por ejemplo: camarón (Márquez-M., 1996) y tiburón (Márquez-M. et al. 2014). La Playa Escobilla se localiza próxima al Golfo de Tehuantepec (GT), donde la 


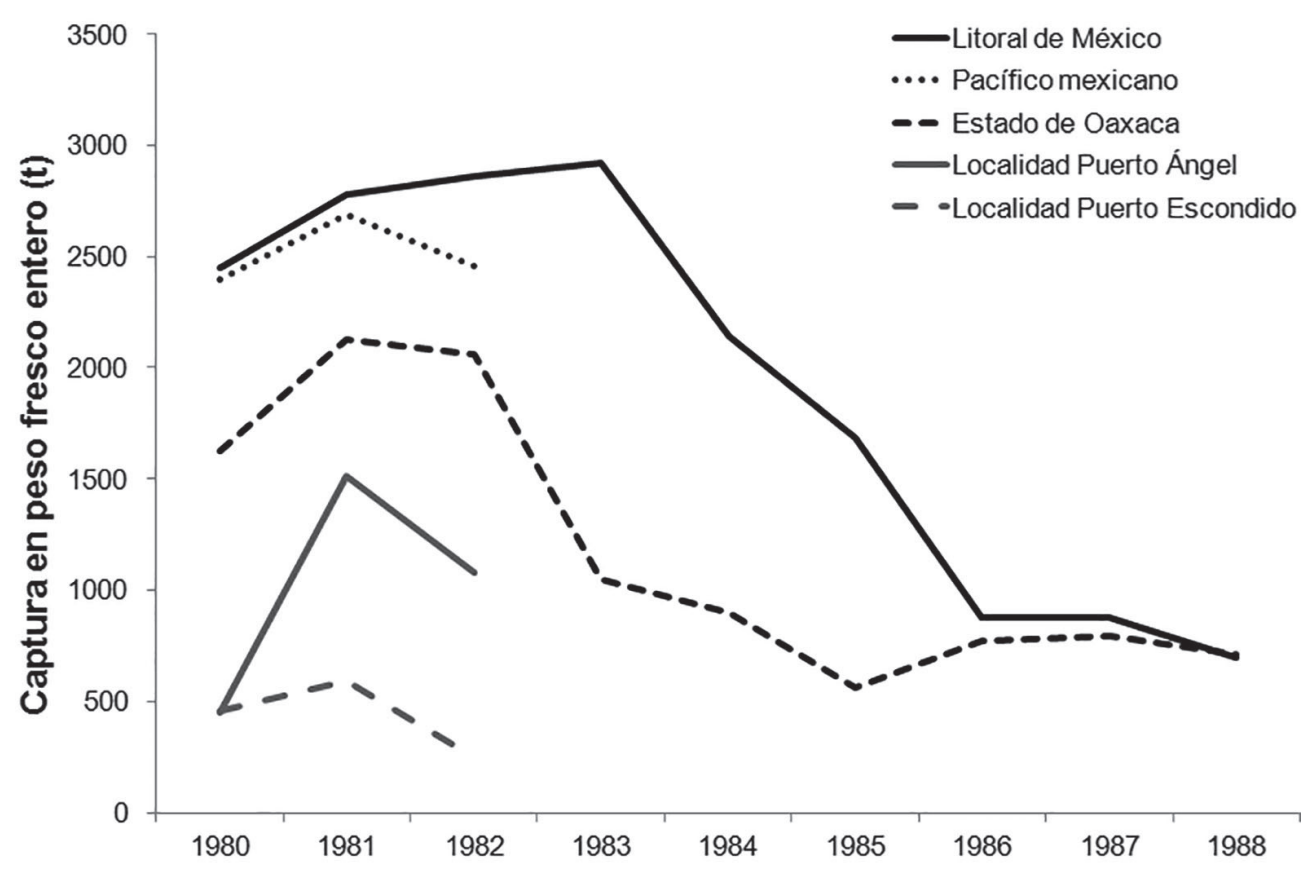

Fig. 1. Registros históricos de captura para la pesquería de tortugas marinas en diferentes regiones y localidades de México

Fig. 1. Historical catch records of marine turtle fishery for different Mexican regions and locations

pesquería del recurso camarón es muy activa y con alcances de actividad hacia los estados de Guerrero y Chiapas. Para la pesquería del recurso camarón del GT, se realizó una extensa revisión de bibliografía y a partir de esta, se discutió sobre su posible relación con la pesquería de Lepidochelys olivacea (Eschscholtz 1829) en la Playa Escobilla. Esta relación se explicó en términos de explotación, por lo que antes de generar la discusión, se realizó la evaluación del estado de explotación para la pesquería de L. olivacea. La Playa Escobilla fue seleccionada de entre otras playas con alta actividad de arribada en el estado de Oaxaca, México (por ejemplo: San
Agustinillo, Morro Ayuta, Chacahua y Barra de la Cruz) (Márquez-M. et al. 1982; Márquez-M., 2014; MerazHernando \& Ávila-Barrientos, 2007), porque únicamente para esta (Fig. 2), está disponible un registro mensual ininterrumpido de captura artesanal.

\section{MATERIALES Y MÉTODOS}

Para realizar la evaluación del estado de explotación, se utilizaron los registros mensuales de captura ribereña con separación entre machos $(\mathrm{Cm}$, en $\mathrm{Kg}$ ) y hembras (Ch, en $\mathrm{Kg}$ ), entre enero de 1978 y mayo de 1990. Estos registros fueron recopilados enteramente por el Centro Mexicano de la Tortuga, Mazunte, Oaxaca, México (Comisión Nacional 
de Áreas Naturales Protegidas). El procesado de los registros se realizó en la Universidad del Mar, Puerto Ángel, Oaxaca. El resumen de esta información se muestra en el Cuadro 1.

\section{Análisis de la mortalidad}

Mortalidad total (Z). Para estimar esta tasa con base mensual, las columnas $\mathrm{Cm}$ y $\mathrm{Ch}$ (Cuadro 1) fueron desplegadas por series de tiempo para identificar a lo largo de cada una de estas, $i$ intervalos de tiempo $>3$ meses, en los que ocurrieron descensos consecutivos para ambos tipos de captura. A cada uno de los $i$ intervalos identificados, se les aplicó el modelo de Berry (1967): $\ln (\mathrm{Cm})$ o $\ln (\mathrm{Ch})$ $=a-\left(\mathrm{Z}_{i} \cdot n\right)($ Ecuación 1) $[a$ es la ordenada al origen, $n$ es el número de descensos que registraron $\mathrm{Cm}$ y $\mathrm{Ch}$ en cada $i$ intervalo de tiempo]. Con base en este proceso se estimaron $Z_{i}$ tasas $\mathrm{y}$, finalmente, se calculó el promedio para machos y hembras.

Mortalidad natural(M). Para estimar esta tasa con base mensual, las columnas $\mathrm{Cm}$ y Ch (Cuadro 1) se ordenaron de manera descendente, para ajustar el modelo exponencial negativo: $\mathrm{NCm}$ o $\mathrm{NCh}=\mathrm{N}_{\mathrm{o}}$ - $\mathrm{e}^{-\mathrm{M}^{*} t}$ (Haddon, 2011) (Ecuación 2) $\left[\mathrm{N}_{\mathrm{o}}\right.$ es el máximo valor de $\mathrm{Cm}$ y Ch extraído durante el período en estudio, $t$ es el tiempo en meses, $\mathrm{NCm}$ y $\mathrm{NCh}$ son los registros esperados para $\mathrm{Cm}$ y $\mathrm{Ch}$.

Los parámetros No y $\mathrm{M}$ fueron estimados con el método Bayesiano de verosimilitud, con el error de estimación ajustado a la distribución log-normal (Haddon, 2011). Al ajustar por primera

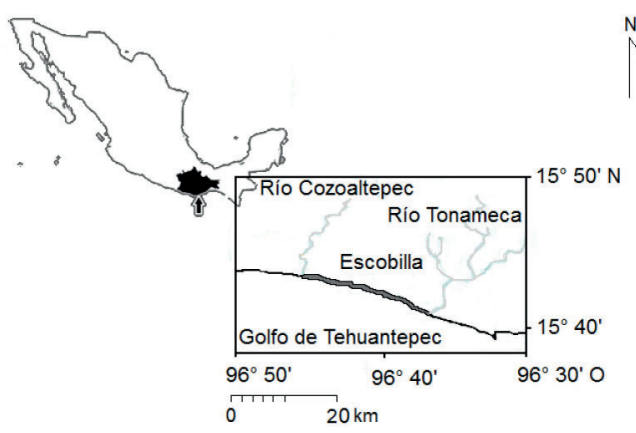

Fig. 2. Localización geográfica de la Playa Escobilla en el estado de Oaxaca, México Fig. 2. Geographic location of Escobilla beach in the state of Oaxaca, Mexico

vez el modelo exponencial negativo, se estimaron ambos parámetros para machos y hembras. Concentrando la atención en el parámetro $\mathrm{M}$, las primeras estimaciones resultantes no fueron tomadas en cuenta, porque de acuerdo con Ricker (1975), se estaría suponiendo que estas tasas fueron constantes en la Playa Escobilla durante el período analizado. Por esta razón y con base en Haddon (2011), se procedió a simular un rango mensual de variación más confiable para las tasas $\mathrm{M}$; para ello, las columnas $\mathrm{Cm}$ y Ch (Cuadro 1), se remuestrearon con remplazo 1000 veces usando la técnica de Bootstrap. Con la técnica Monte Carlo, el modelo exponencial negativo se ajustó a cada remuestreo, generando 1000 estimaciones de la tasa M para machos y hembras. Posteriormente, para cada sexo se implementaron dos análisis por histogramas de frecuencias (Zar, 1999). Con esto último, los valores estimados de las tasas $\mathrm{M}$ fueron analizados con base en su frecuencia de repetición y tomando en cuenta las 
Cuadro 1. Registros de captura para Lepidochelys olivacea en la Playa Escobilla, Oaxaca, México, período enero 1978 - mayo 1990. Captura machos (Cm), Captura hembras $(\mathrm{Ch})$, Captura Total $\left(\mathrm{C}_{\mathrm{T}}=\mathrm{Cm}+\mathrm{Ch}\right)$

Table 1. Catch records for Lepidochelys olivacea at Escobilla beach, Oaxaca, Mexico, January 1978 - May 1990 period. Male Catch $(\mathrm{Cm})$, Female Catch $(\mathrm{Ch})$, Total Catch $\left(\mathrm{C}_{\mathrm{T}}=\mathrm{Cm}+\mathrm{Ch}\right)$

\begin{tabular}{|c|c|c|c|c|c|c|c|c|c|c|c|c|c|c|c|c|c|c|c|}
\hline Años & Meses & $\mathrm{Cm}$ & $\mathrm{Ch}$ & $\mathrm{C}_{\mathrm{T}}$ & & & $\mathrm{Cm}$ & $\mathrm{Ch}$ & $\mathrm{C}_{\mathrm{T}}$ & & & $\mathrm{Cm}$ & $\mathrm{Ch}$ & $\mathrm{C}_{\mathrm{T}}$ & & & $\mathrm{Cm}$ & $\mathrm{Ch}$ & $\mathrm{C}_{\mathrm{T}}$ \\
\hline \multirow[t]{12}{*}{1978} & 1 & 44 & 271 & 315 & 1982 & 1 & 2601 & 289 & 2890 & 1986 & 1 & 29 & 527 & 29 & 1990 & 1 & 10 & 308 & 318 \\
\hline & 2 & 91 & 434 & 525 & & 2 & 717 & 107 & 824 & & 2 & 103 & 391 & 103 & & 2 & 5 & 10 & 15 \\
\hline & 3 & 362 & 389 & 751 & & 3 & 865 & 149 & 1014 & & 3 & 306 & 281 & 306 & & 3 & 30 & 31 & 61 \\
\hline & 4 & 195 & 123 & 318 & & 4 & 1023 & 668 & 1691 & & 4 & 564 & 269 & 564 & & 4 & 7 & 9 & 16 \\
\hline & 5 & 236 & 1481 & 1717 & & 5 & 1243 & 595 & 1838 & & 5 & 336 & 395 & 336 & & 5 & 80 & 44 & 124 \\
\hline & 6 & 278 & 2838 & 3116 & & 6 & 29 & 45 & 74 & & 6 & 255 & 363 & 255 & & & & & \\
\hline & 7 & 319 & 4196 & 4515 & & 7 & 42 & 2639 & 2681 & & 7 & 174 & 332 & 174 & & & & & \\
\hline & 8 & 175 & 7473 & 7648 & & 8 & 45 & 4089 & 4134 & & 8 & 93 & 300 & 93 & & & & & \\
\hline & 9 & 130 & 9033 & 9163 & & 9 & 5 & 5952 & 5957 & & 9 & 12 & 268 & 12 & & & & & \\
\hline & 10 & 33 & 4433 & 4466 & & 10 & 9 & 6579 & 6588 & & 10 & 27 & 4173 & 27 & & & & & \\
\hline & 11 & 367 & 14616 & 14983 & & 11 & 69 & 5934 & 6003 & & 11 & 27 & 2661 & 27 & & & & & \\
\hline & 12 & 2418 & 9629 & 12047 & & 12 & 43 & 115 & 158 & & 12 & 32 & 353 & 32 & & & & & \\
\hline \multirow[t]{12}{*}{1979} & 1 & 421 & 1020 & 1441 & 1983 & 1 & 363 & 178 & 541 & 1987 & 1 & 7 & 460 & 7 & & & & & \\
\hline & 2 & 139 & 151 & 290 & & 2 & 683 & 240 & 923 & & 2 & 24 & 347 & 24 & & & & & \\
\hline & 3 & 121 & 279 & 400 & & 3 & 1003 & 303 & 1306 & & 3 & 84 & 159 & 84 & & & & & \\
\hline & 4 & 46 & 71 & 117 & & 4 & 1323 & 365 & 1688 & & 4 & 201 & 309 & 201 & & & & & \\
\hline & 5 & 839 & 806 & 1645 & & 5 & 383 & 507 & 890 & & 5 & 297 & 293 & 297 & & & & & \\
\hline & 6 & 279 & 1567 & 1846 & & 6 & 58 & 122 & 180 & & 6 & 156 & 492 & 156 & & & & & \\
\hline & 7 & 193 & 2361 & 2553 & & 7 & 20 & 1904 & 1924 & & 7 & 14 & 690 & 14 & & & & & \\
\hline & 8 & 106 & 3154 & 3260 & & 8 & 19 & 3692 & 3711 & & 8 & 42 & 2102 & 42 & & & & & \\
\hline & 9 & 89 & 3083 & 3172 & & 9 & 77 & 3191 & 3268 & & 9 & 6 & 2016 & 6 & & & & & \\
\hline & 10 & 42 & 895 & 937 & & 10 & 95 & 3222 & 3317 & & 10 & 23 & 1918 & 23 & & & & & \\
\hline & 11 & 201 & 5567 & 5768 & & 11 & 157 & 1762 & 1919 & & 11 & 26 & 2145 & 26 & & & & & \\
\hline & 12 & 455 & 5116 & 5571 & & 12 & 98 & 777 & 875 & & 12 & 20 & 908 & 20 & & & & & \\
\hline \multirow[t]{12}{*}{1980} & 1 & 446 & 1616 & 2062 & 1984 & 1 & 106 & 552 & 658 & 1988 & 1 & 23 & 528 & 23 & & & & & \\
\hline & 2 & 356 & 572 & 928 & & 2 & 349 & 615 & 964 & & 2 & 46 & 197 & 46 & & & & & \\
\hline & 3 & 518 & 810 & 1328 & & 3 & 588 & 554 & 1142 & & 3 & 58 & 44 & 58 & & & & & \\
\hline & 4 & 508 & 416 & 924 & & 4 & 778 & 386 & 1164 & & 4 & 335 & 190 & 335 & & & & & \\
\hline & 5 & 796 & 573 & 1369 & & 5 & 627 & 465 & 1092 & & 5 & 221 & 210 & 221 & & & & & \\
\hline & 6 & 429 & 1091 & 1520 & & 6 & 364 & 674 & 1038 & & 6 & 194 & 590 & 194 & & & & & \\
\hline & 7 & 61 & 1609 & 1670 & & 7 & 100 & 883 & 983 & & 7 & 166 & 971 & 166 & & & & & \\
\hline & 8 & 177 & 2741 & 2918 & & 8 & 89 & 1447 & 1536 & & 8 & 139 & 1351 & 139 & & & & & \\
\hline & 9 & 20 & 2127 & 2147 & & 9 & 458 & 1237 & 1695 & & 9 & 46 & 1969 & 46 & & & & & \\
\hline & 10 & 233 & 2856 & 3089 & & 10 & 243 & 1692 & 1935 & & 10 & 14 & 2148 & 14 & & & & & \\
\hline & 11 & 37 & 4688 & 4725 & & 11 & 90 & 1598 & 1688 & & 11 & 1 & 1919 & 1 & & & & & \\
\hline & 12 & 155 & 3443 & 3598 & & 12 & 51 & 804 & 855 & & 12 & 16 & 1335 & 16 & & & & & \\
\hline \multirow[t]{12}{*}{1981} & 1 & 658 & 715 & 1373 & 1985 & 1 & 109 & 487 & 595 & 1989 & 1 & 19 & 561 & 19 & & & & & \\
\hline & 2 & 444 & 474 & 918 & & 2 & 166 & 169 & 335 & & 2 & 99 & 145 & 99 & & & & & \\
\hline & 3 & 276 & 313 & 589 & & 3 & 215 & 154 & 369 & & 3 & 338 & 153 & 338 & & & & & \\
\hline & 4 & 149 & 293 & 442 & & 4 & 368 & 119 & 487 & & 4 & 450 & 253 & 450 & & & & & \\
\hline & 5 & 492 & 631 & 1123 & & 5 & 262 & 122 & 384 & & 5 & 317 & 379 & 317 & & & & & \\
\hline & 6 & 296 & 1079 & 1375 & & 6 & 209 & 1060 & 1269 & & 6 & 213 & 609 & 213 & & & & & \\
\hline & 7 & 99 & 1527 & 1626 & & 7 & 156 & 1999 & 2155 & & 7 & 110 & 839 & 110 & & & & & \\
\hline & 8 & 73 & 3610 & 3683 & & 8 & 103 & 2937 & 3040 & & 8 & 6 & 1069 & 6 & & & & & \\
\hline & 9 & 70 & 4154 & 4224 & & 9 & 345 & 3309 & 3654 & & 9 & 15 & 1714 & 15 & & & & & \\
\hline & 10 & 65 & 5823 & 5888 & & 10 & 830 & 6139 & 6969 & & 10 & 6 & 2100 & 6 & & & & & \\
\hline & 11 & 162 & 6234 & 6396 & & 11 & 282 & 3177 & 3459 & & 11 & 6 & 1563 & 6 & & & & & \\
\hline & 12 & 1465 & 2155 & 3620 & & 12 & 115 & 1290 & 1405 & & 12 & 31 & 689 & 31 & & & & & \\
\hline
\end{tabular}


más altas, se implementaron los rangos mensuales de variación que incluyeron a los valores máximos y mínimos de la tasa $\mathrm{M}$ ocurridos entre enero de $1978 \mathrm{y}$ mayo de 1990.

\section{Mortalidad por pesca ribereña}

(F). Para estimar esta tasa con base mensual, se aplicó el modelo $\mathrm{F}=\mathrm{Z}-\mathrm{M}$ (Beverton \& Holt, 1957), cumpliendo con el supuesto de que $\mathrm{M}<\mathrm{Z}$. Lo anterior se resolvió para machos y hembras, usando los respectivos valores estimados de las tasas Z y M. La tasa de explotación (E) se estimó con $\mathrm{E}=\mathrm{F} / \mathrm{Z}$ (Ricker, 1975).

En el presente estudio, el parámetro no poblacional $a$ en la Ecuación 1 y el parámetro poblacional No en la Ecuación 2 no fueron interpretados. Para el caso de No, esto fue porque en el Pacífico mexicano, la pesquería de tortugas marinas comenzó desde 1959 (Márquez-M. et al. 1982; Trinidad \& Wilson, 2000) y no desde enero del año 1978. El análisis de mortalidad fue realizado con el programa PopTool ${ }^{\odot}$ versión 2.7.5 (Hood, 2006).

\section{Series de tiempo para la captura ribereña}

\section{Tendencias netas}

Para las columnas $\mathrm{Cm}$ y $\mathrm{Ch}$ (Cuadro 1), se simularon las "tendencias netas" con el método Smoothed trend CENSUS I (StatSoft, 2002). En relación con los tipos de explotación documentados por Beverton \& Holt (1957), para este trabajo, las tendencias netas se consideraron como: "subexplotado" (las tendencias netas de $\mathrm{Cm}$ y $\mathrm{Ch}$ son ascendentes), "sobreexplotado" (las tendencias netas de $\mathrm{Cm}$ y $\mathrm{Ch}$ son descendentes) y en "equilibrio"
Cuadro 2. Parámetros estimados de explotación para Lepidochelys olivacea en la Playa Escobilla, período enero 1978 - mayo 1990. Promedios mensuales para las tasas de mortalidad total $(\mathrm{Z})$, natural promedio (M), por pesca artesanal $(\mathrm{F})$ y explotación (E). Machos $\left({ }_{m}\right)$ y hembras $\left({ }_{h}\right)$ (ver texto para la explicación)

Table 2. Estimated exploitation parameters of Lepidochelys olivacea at Escobilla beach, January 1978 - May 1990 period. Monthly averages of total mortality (Z), natural mortality (M), fishing mortality (F) and exploitation (E) rates. Male $\left({ }_{\mathrm{m}}\right)$ and female $(\mathrm{h})$ (see text for explanation)

\begin{tabular}{ccccc}
\hline & $\mathbf{Z}$ & $\mathbf{M}$ & $\mathbf{F}$ & $\mathbf{E}$ \\
\hline machos & $0.29_{(\mathrm{m})}$ & 0.03 & 0.26 & 0.90 \\
hembras & $0.38_{(\mathrm{h})}$ & 0.04 & 0.34 & 0.90 \\
\hline
\end{tabular}

(las tendencias netas de $\mathrm{Cm}$ y $\mathrm{Ch}$ no tienen cambios claros ascendentes o descendentes). Para explicar los tipos de explotación, se utilizaron los valores estimados de las tasas Z, F, M y E.

\section{Temporadas de arribada}

Otro análisis se realizó a las columnas $\mathrm{Cm}$ y $\mathrm{Ch}$ (Cuadro 1), estas fueron adicionadas para obtener la captura total mensual ribereña o $\mathrm{C}_{\mathrm{T}}$ (Cuadro 1). Para esta última se simuló el "comportamiento armónico mensual (CAm)" con el método Seasonal Factor CENSUS I (StatSoft, 2002). El CAm es una serie de tiempo cíclica (una transformación de la original $\mathrm{C}_{\mathrm{T}}$ ), constituida por anomalías mensuales positivas y negativas, con el valor de cero como punto medio de cambio. Para este trabajo y siguiendo a Makridakis et al. (1983) y Cervantes-Hernández et al. (2016), los incrementos mensuales 

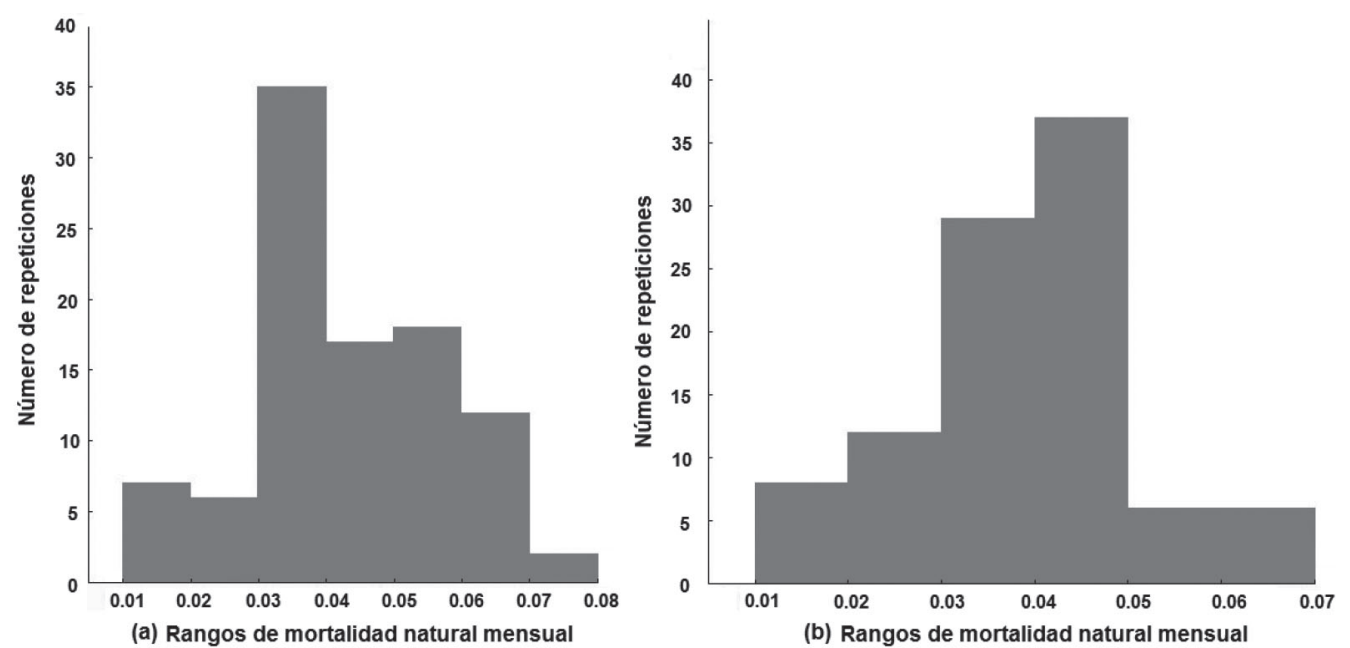

Fig. 3. Rangos de variación mensual para la tasa de mortalidad natural (M) en la población de Lepidochelys olivacea, período enero 1978 - mayo 1990. Machos (a) y hembras (b) Fig. 3. Monthly variation ranges for natural mortality rate (M) in the population of Lepidochelys olivacea, January 1978 - May 1990 period. Male (a) and female (b)

de $\mathrm{C}_{\mathrm{T}}$ se representaron con las anomalías positivas y los decrementos mensuales se representaron con anomalías negativas. Con el CAm, se lograron acotar el inicio, el máximo y el fin de las temporadas de arribada de L. olivacea. Para el análisis del CAm, se utilizó la $\mathrm{C}_{\mathrm{T}}$, porque de acuerdo con Márquez-M. et al. (1982) y MerazHernando \& Ávila-Barrientos (2007), en una misma temporada de arribada, los machos y las hembras coinciden espacialmente en las mismas playas de anidación, para realizar repetidos ciclos de desove y anidación.

\section{RESULTADOS}

\section{Análisis de la mortalidad}

Para machos (Cuadro 2), la tasa $\mathrm{M}$ resultó con un rango de variación de 0.03 a 0.04 mensual (Shapiro-Wilks $W=0.959, P<0.05)$, con una frecuencia del 23\% para la repetición del intervalo (Fig. 3). Para hembras (Cuadro 2), esta tasa tuvo un rango de variación de 0.04 a 0.05 mensual $(W=0.974, P$ $<0.05$ ), con una frecuencia del 25\% en la repetición del intervalo (Fig. 3). Estos resultados indicaron, que en la Playa Escobilla, la tasa M tuvo una baja afectación sobre la población de L. olivacea, porque en promedio tres machos y cuatro hembras de cada 100 , murieron al mes por causas naturales. Para el modelo $\mathrm{F}=\mathrm{Z}-\mathrm{M}$, se cumplió que $\mathrm{M}<\mathrm{Z}$ y en comparación con las tasas M, las afectaciones de la tasa F resultaron mayores, porque 26 machos y 34 hembras de cada 100, murieron mensualmente por causas asociadas a la pesca artesanal.

Aunque la tasa $\mathrm{F}$ resultó mayor para hembras con respecto a machos, se demostró estadísticamente, que ambos sexos fueron explotados con la 
misma intensidad, porque al comparar las tasas $\mathrm{Z}$ de hembras $\left(_{\mathrm{h}}\right)\left(\mathrm{Z}_{(\mathrm{h})}=0.38\right.$, $\left.\sigma_{2}=0.17, \mathrm{~N}_{1}=51\right)$ y machos $\left(_{\mathrm{m}}\right)\left(\mathrm{Z}_{(\mathrm{m})}\right.$ $=0.29, \sigma_{1}=0.11, \mathrm{~N}_{1}=59$ ) (Cuadro 2), la hipótesis nula fue aceptada (Zar, 1999); esto es, ambas tasas $Z$ son aproximadamente iguales (distribución $\left.Z=0.76, Z_{0.05}=1.65, P>0.05\right)$. Esta igualdad se confirmó por segunda ocasión al comparar directamente los valores de las tasas E (Cuadro 2).

\section{Series de tiempo para la captura ribereña}

\section{Tendencias netas}

Las tendencias netas que resultaron para $\mathrm{Cm}$ y $\mathrm{Ch}$ fueron descendentes (Fig. 4) y con base en los resultados del Cuadro 2, se corroboró que ambos sexos de L. olivacea fueron sobreexplotados con la misma intensidad durante el período en estudio.

\section{Temporadas de arribada}

El CAm para $\mathrm{C}_{\mathrm{T}}$ se ilustra en la Figura 5. El CAm resultó con escala de 1 a 25 para las anomalías positivas y entre -1 y -15 para las anomalías negativas. Con el seguimiento de las anomalías positivas, se evidenció que la temporada de arribada de L. olivacea en la Playa Escobilla, transcurrió interanualmente de agosto a enero, con el máximo de arribada en noviembre. Un total de 12 temporadas de arribada se registraron en el período de estudio.

\section{DISCUSIÓN}

En la dinámica de poblaciones explotadas, la estimación correcta de la tasa M es importante (Vetter, 1988), porque con esta se diferencian las muertes naturales, de las ocasionadas por la acción de pesca en $\mathrm{Z}=\mathrm{M}+\mathrm{F}$ (Beverton \& Holt, 1957; Ramos-Cruz et al. 2006; Cervantes-Hernández \& Gracia, 2011). Nunca antes las tasas Z, $\mathrm{M}, \mathrm{F}$ y $\mathrm{E}$ fueron estimadas para ninguna de las poblaciones de tortugas marinas en el Pacífico mexicano; y en particular, en la Playa Escobilla. Para el análisis de la mortalidad, se utilizaron los modelos más confiables recomendados en la literatura (Ramos-Cruz et al. 2006; Cervantes-Hernández \& Gracia, 2011; Cervantes-Hernández et al. 2016).

En las tortugas marinas, las afectaciones de la mortalidad natural varían a lo largo de la historia de vida, siendo mayores en magnitud durante los estadios de huevo/cría (por ejemplo: de 10000 crías solo el $0.2 \%$ alcanzan la edad adulta) (Hirth \& Schafter, 1974; Hughes \& Richard, 1974; Márquez-M. et al. 1976; Seminoff et al. 2008) y disminuyen al incrementarse la talla (Márquez-M., 1996).

En la Playa Escobilla, se demostró que la población de L. olivacea estuvo sobreexplotada de enero del año 1978 a mayo del año 1990. Lo anterior se explicó con las tendencias netas descendentes de $\mathrm{Cm}$ y Ch (Fig. 4), que fueron similares a las mostradas en la Figura 1. Durante el transcurso del proceso de sobreexplotación, la tasa $\mathrm{F}$ se mantuvo mayor a la tasa $\mathrm{M}$; y la afectación de esta última resultó baja para esta población (Cuadro 2). Como resultado de esto, ambos sexos fueron explotados con la misma intensidad 


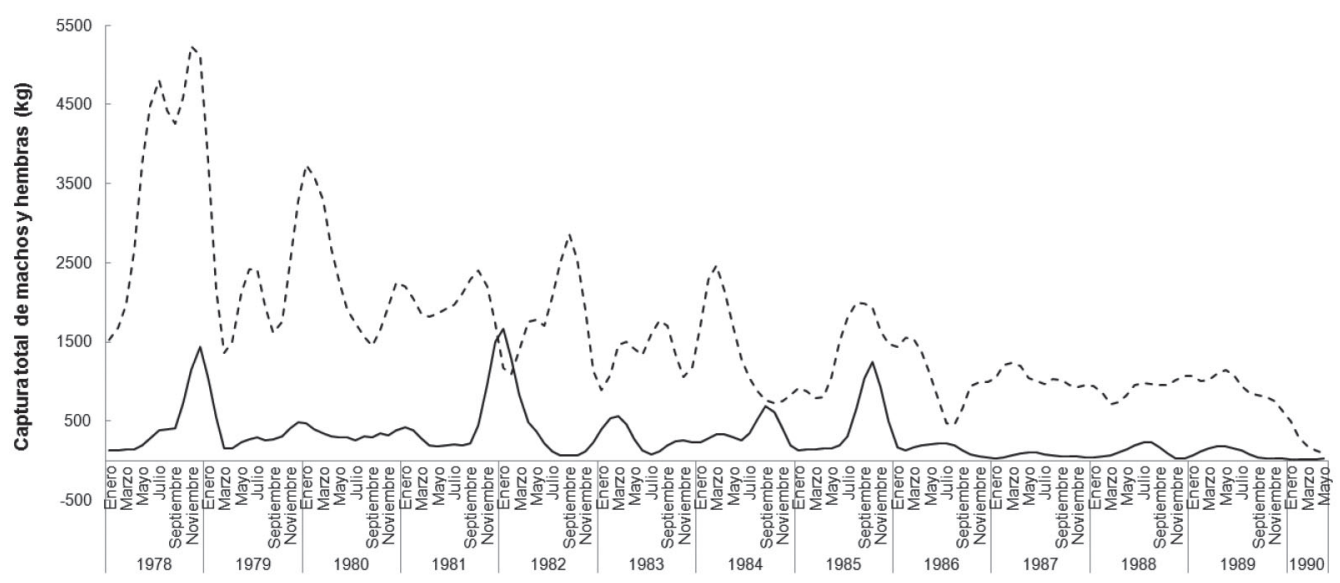

Fig. 4. Tendencias históricas de captura para los machos (línea negra continua) y las hembras (línea negra punteada) de Lepidochelys olivacea en la Playa Escobilla, período enero 1978 - mayo 1990

Fig. 4. Historical catch trends for male (continuous black line) and female (discontinuous gray line) of Lepidochelys olivacea at Escobilla beach, January 1978 - May 1990 period

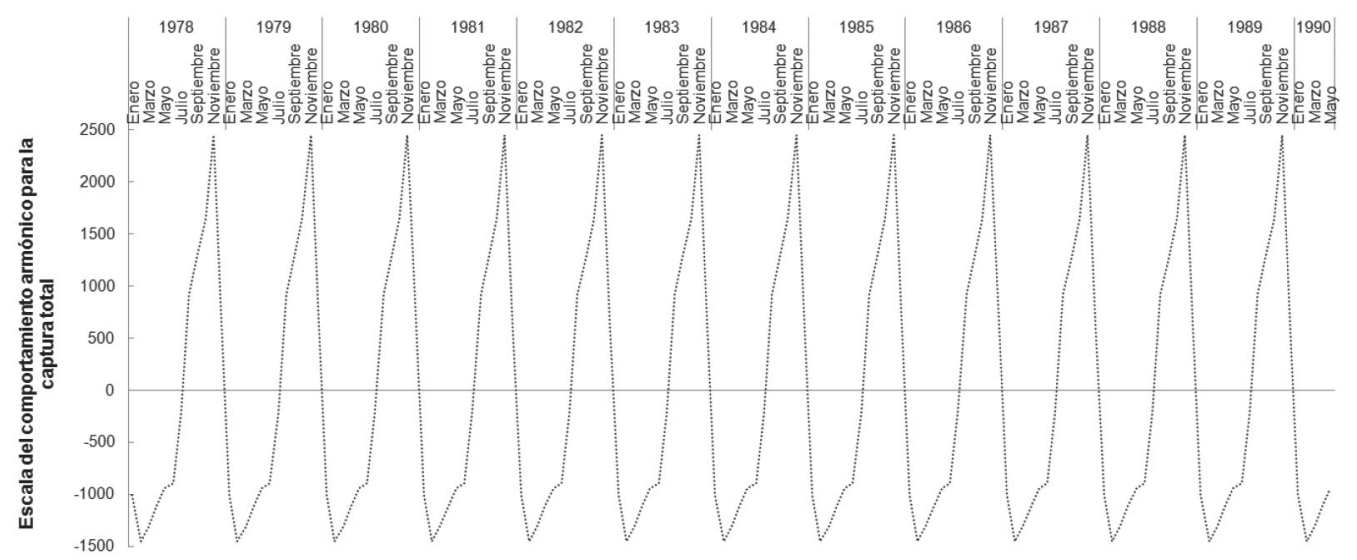

Fig. 5. Comportamiento armónico mensual de la captura total para Lepidochelys olivacea en la Playa Escobilla, período enero 1978 - mayo 1990. Las temporadas de arribada están indicadas con anomalías mensuales positivas

Fig. 5. Monthly harmonic behavior of total catch of Lepidochelys olivacea at Escobilla beach, January 1978 - May 1990 period. Nesting seasons are indicated with positive monthly anomalies

(Cuadro 2). A partir de lo anterior y de acuerdo con Márquez-M. (1996), se infirió que en la Playa Escobilla la captura ribereña pudo haber estado integrada por ejemplares adultos con tallas comerciales $>52 \mathrm{~cm}$.

La temporada de arribada de L. olivacea transcurrió de agosto a enero, con 
el máximo en noviembre (Fig. 5). Para las costas de Oaxaca, está documentado que esta es de julio a diciembre con extensión hasta enero (MárquezM., 1996); y adicionalmente, MerazHernando \& Ávila-Barrientos (2007) mencionaron que el máximo de arribada ocurre entre octubre y noviembre, con disminución hacia la primavera. La temporada de arribada que resultó en este trabajo (Fig. 5) coincidió parcialmente al inicio y al final con MárquezM. (1996) ( \pm 1 meses); pero el máximo coincidió con Meraz-Hernando \& Ávila-Barrientos (2007) (Fig. 5). Para el estado de Michoacán, México, García-Romero et al. (2006) documentaron la temporada de arribada coincidiendo completamente con la Figura 5. Al considerar el sustento bibliográfico, se consideró que los resultados obtenidos para las temporadas de arribada, son confiables como puntos de referencia para el uso y el aprovechamiento actuales de las tortugas marinas en el Pacífico mexicano y en la Playa Escobilla.

Margaleff (1978) documentó que los incrementos en la abundancia y la agregación poblacionales tienen una relación directa con la intensidad reproductiva. Esto sugiere, que el transcurso de la temporada de arribada aquí descrita (Fig. 5), funcionó como un indicador para validar la variación mensual de la abundancia de L. olivacea. Sin embargo, está considerado que esta información ya formaba parte del conocimiento empírico por parte de los usuarios de esta pesquería, porque ellos reconocen, para sincronizar su pesca, las fechas de máxima y mínima disponibilidad del recurso.

Para el manejo de la pesquería del recurso del camarón en todo el Pacífico mexicano, se han implantado diferentes temporadas de veda, estas fueron: de julio a septiembre entre 1979 y 1987 (Fig. 6), de mayo/abril a septiembre de 1993 a 1997 (Cervantes-Hernández et al. 2008a), de abril a septiembre en 1999; y posterior al 2001, entre marzo/abril y septiembre (INAPESCA, 2007a; 2007b). Para el caso específico del "camarón café" Farfantepenaeus californiensis (Holmes 1900) del GT, los primeros autores demostraron con un CAm que la temporada reproductiva de $F$. californiensis transcurre de julio a febrero, con la mayor actividad de desove entre los meses de octubre y noviembre (Fig. 6). La efectividad de las temporadas de veda en el GT ha sido insuficiente, porque estas han protegido por completo a las temporadas de reclutamiento y no a las temporadas reproductivas. Esta última temporada ha transcurrido en pleno durante las temporadas oficiales de pesca, que para el GT son de septiembre/octubre a marzo/abril (INAPESCA, 2007a; 2007b).

Para el GT, Cervantes-Hernández et al. (2008b) demostraron que las temporadas reproductivas de $F$. californiensis se desarrollan recurrentes en condiciones oceanográficas de alta concentración de clorofila $a \quad(\mathrm{R}=0.562, P<0.05)$ y en un entorno frío para la temperatura superficial del mar $(\mathrm{R}=-0.597, P<0.05)$. Fernández-Álamo et al. (2000) señalaron que incrementos en clorofila $a$ propician el aumento en la disponibilidad de alimento 


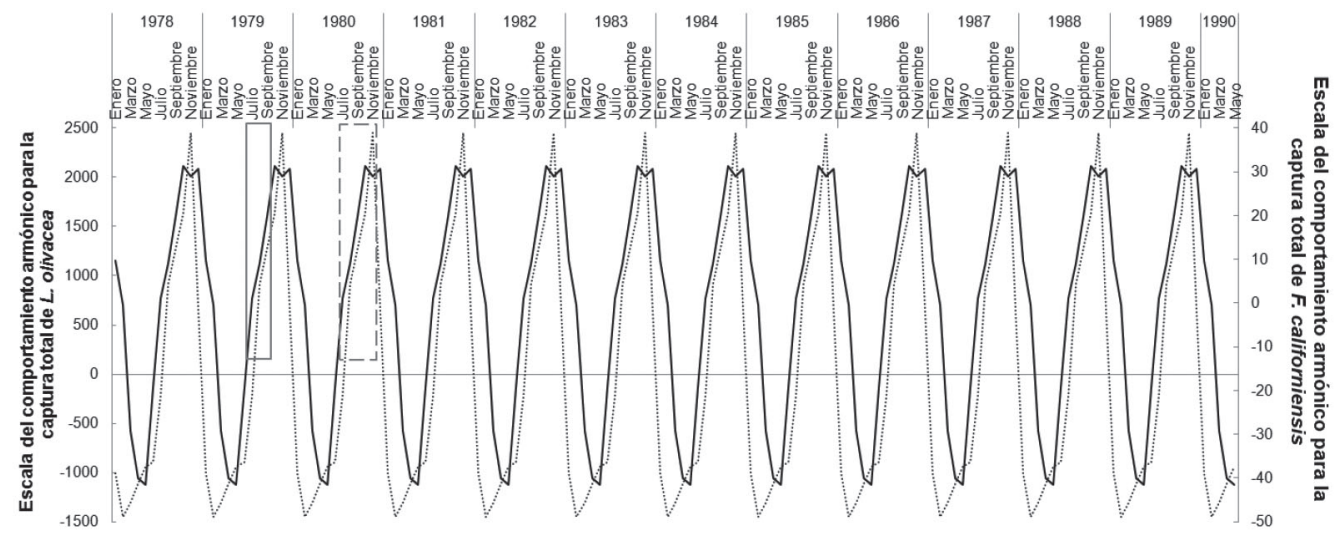

Fig. 6. Comportamientos armónicos mensuales de captura en las pesquerías de Lepidochelys olivacea (línea punteada negra) y Farfantepenaeus californiensis del Golfo de Tehuantepec (línea continua negra) (Cervantes-Hernández et al. 2008a). Las temporadas de arribada están indicadas con anomalías mensuales positivas. Para el recurso camarón del Pacífico mexicano, la temporada de veda más antigua registrada es de junio/julio a octubre (rectángulo gris continuo) (INAPESCA, 2007a; 2007b). Temporada de veda propuesta para proteger a las poblaciones de tortuga y de camarón de julio a noviembre (rectángulo gris discontinuo)

Fig. 6. Monthly harmonic behavior of catch in the fisheries of Lepidochelys olivacea (gray dotted line) and Farfantepenaeus californiensis at the Gulf of Tehuantepec (continuous black line) (Cervantes-Hernández et al. 2008a). Nesting seasons are indicated with positive monthly anomalies. For the Mexican Pacific shrimp, the earliest close season recorded was from June/July to October (continuous gray rectangle) (INAPESCA, 2007a; 2007b). Close season proposed to protect turtle and shrimp populations from July to November (discontinuous gray rectangle)

(biomasa del zooplancton); que a su vez, aumenta la probabilidad de supervivencia para los estadios larvarios de F. californiensis (protozoea/mysis), amplificando la cadena trófica. Cervantes-Hernández (2015) reportó las mismas condiciones oceanográficas en el Golfo de México, al transcurrir la temporada reproductiva del "camarón café" Farfantepenaeus aztecus (Ives 1891), de noviembre a agosto, con la mayor actividad de desove entre los meses de febrero y marzo.

Al comparar los resultados de Cervantes-Hernández et al. (2008a; 2008b) y los obtenidos con el CAm (Fig. 6), se encontró que las temporadas reproductivas de $F$. californiensis y de arribada para L. olivacea, coincidieron desarrollándose casi sincrónicamente. Esto tiene relación con las condiciones oceanográficas imperantes en el GT (y áreas adyacentes, la Playa Escobilla) $y$, particularmente, en lo que respecta a los máximos de noviembre, cuando el suministro de alimento se maximiza (Fernández-Álamo et al. 2000; Cervantes-Hernández et al. 2008b). El incremento en la cadena trófica durante el 
transcurso de la temporada de "Tehuanos" representa una ventaja alimenticia para la arribada de L. olivacea.

En la región del GT, las temporadas de Tehuanos son de noviembre a febrero (Fernández-Álamo et al. 2000) y se caracterizan por el desarrollo de "surgencias" eólicas, que enriquecen los estratos superficiales oceánicos con nutrientes provenientes del fondo frío (Gallegos-García \& BarberánFalcón, 1998), generando cambios en la concentración de clorofila $a$ y la producción primaria. Fuera de esta temporada, el GT funciona como un ecosistema tropical con valores bajos de clorofila a (Lara-Lara et al. 1998), donde la producción primaria es compensada localmente por la precipitación pluvial y la descarga fluvial (Frías-Velasco, 2004).

Está documentada, pero no corroborada, que la sobreexplotación de tortugas marinas en el Pacífico mexicano fue provocada por el exceso de captura incidental que ocasionó la pesca de camarón (Márquez-M., 1996). Para el GT, esto puede explicarse parcialmente, si se toma en cuenta que durante las temporadas reproductivas de $F$. californiensis, las actividades de pesca se incrementan gradualmente, generando mayor captura incidental para las especies cuya historia de vida coincida con la temporada de pesca de la primera (caso L. olivacea, Fig. 6). Existen evidencias aisladas que demuestran que esto pudo ocurrir, porque anterior al año de 1996, los excluidores de tortugas no fueron reglamentados (SEMARNAP, 1996-
1996); y Tapia-García \& GutiérrezDíaz (1998) reportaron la persistencia de $L$. olivacea en la captura incidental del recurso camarón. Esta misma tendencia está documentada en el Golfo de México, Caillouet et al. (1991) reportaron que durante las maniobras de pesca dirigidas al recurso camarón, muchas tortugas marinas resultaron con mutilaciones y fracturas músculoesqueléticas, ya que son atraídas por los desechos que se generan en las áreas de arrastre. Márquez-M. et al. (1976) mencionaron que en la dieta de L. olivacea está incluido el camarón, pero predominantemente consumen peces y otros crustáceos.

En el presente, la pesquería del recurso camarón continúa realizándose en el Pacífico mexicano, pero desde los años noventa y debido a la mala planeación en sus temporadas de veda, la pesquería se ha mantenido en un nivel crítico de explotación(CervantesHernández et al. 2006). Esta situación, en el GT, aparentemente ha reducido la captura incidental de tortugas marinas en las maniobras de pesca, sin embargo, esto no se ha podido corroborar por la falta de registros en los Anuarios Estadísticos de Pesca. Se propuso que si el recurso camarón del GT no se encontrase como fue señalado por los autores mencionados, en la actualidad, la captura incidental de tortugas marinas en el GT seguiría mermando a esta población como se muestra en las Figuras 1 y 4.

Para extender la protección hacia las temporadas reproductivas de $F$. californiensis y Litopenaeus vanna- 
mei (Boone 1931) del GT, CervantesHernández et al. (2008a) propusieron el reajuste de las temporadas de veda de junio/julio a octubre. Durante el desarrollo de este trabajo, la Figura 6 fue tomada en cuenta para proponer un ajuste diferente, que es entre julio y noviembre. En este rango de tiempo, se continuaría cumpliendo con lo indicado por los autores mencionados $\mathrm{y}$ en un segundo plano, se evitaría coincidir el aumento creciente de las actividades de pesca de camarón, con el transcurso gradual ascendente de las temporadas de arribada (del inicio hasta el máximo). Con esta nueva propuesta, no habría la necesidad de implementar dos vedas por separado ni continuar prolongando la existente para las tortugas marinas. El recurso camarón podría ser explotado sustentablemente, evitando que las tortugas marinas continúen siendo, como lo mencionaron Márquez-M. (1996) y Tapia-García \& Gutiérrez-Díaz (1998). La economía del sector pesquero de camarón resultaría favorecida, porque no habría la necesidad de invertir más en tecnología para mejorar a los excluidores de tortugas, reduciendo gastos innecesarios por sanciones administrativas.

En México, a partir de 1996, la población de L. olivacea en la Playa Escobilla se reportó ascendiendo de 57 mil (Márquez-M. et al. 1982) a cerca de un millón de nidos (Peñaflores-Salazar et al. 2001). En los últimos 12 años y por temporada de arribada, se documentaron más de un millón de nidadas en la Playa
Escobilla (Márquez-M et al. 2014). Para el Pacífico mexicano, Seminoff et al. (2008) reportaron un incremento general en la actividad de anidación.

En los estados de Michoacán y Guerrero, México, están documentadas las mismas tendencias descendentes en la captura de tortugas marinas, como se ilustró en las Figuras 1 y 4. Para Michoacán, la tendencia fue de 452 t en 1980 a $56 \mathrm{t}$ en 1988 y para Guerrero, de 112 t en 1980 a 39 t en 1988 (PESCA, 1980-1989). Para todo el Pacífico mexicano, la información mencionada representa el $48 \%$ del total disponible y el único registro de pesca ribereña ininterrumpido del que se tiene conocimiento fue hecho para L. olivacea en la Playa Escobilla. Por esta razón, los resultados aquí descritos pueden considerarse como confiables, tal y como lo fueron con el CAm para F. californiensis según CervantesHernández et al. (2008a).

Para considerar una planificación futura del uso y manejo de L. olivacea, además de lo mencionado aquí, se debe prestar especial atención a los problemas locales de tipo no pesquero. En la Playa Escobilla, Benabib \& Wilson (1981) reportaron incrementos en la muerte natural, debido a la depredación de embriones y crías eclosionadas por parte del escarabajo Omorgus suberosus (Fabricius, 1775). Enciso-Saracho (2007) reportó el aumento de ejemplares arribados con anormalidades anatómicas, parálisis, tumores (fibropapilomas) y ectoparasitosis.

Otro aspecto importante es el control de la mortalidad natural 
denso-dependiente a nivel de playa. Márquez-M. (1996) documentó y comparó este tipo de afectación entre las poblaciones de L. olivacea de las playas Escobilla y Nancite, Santa Rosa, Costa Rica. Aun cuando para estas dos localidades geográficas está documentado un esfuerzo reproductor similar, los resultados en el éxito de anidación e incubación fueron muy distintos. Lo anterior fue fundamentado refiriéndose a la Playa Escobilla como muy amplia y recta, con más de $7 \mathrm{~km}$ de largo (Fig. 2); mientras que la Playa Nancite es estrecha y de $1 \mathrm{~km}$ (Hughes \& Richard, 1974). Cuando ocurre la mayor arribada en la Playa Escobilla (Fig. 5), el espacio disponible para anidar es 7 veces más amplio; y por lo tanto, la mortalidad natural denso-dependiente resulta menor en comparación con la playa costarricense. Entre las causas que incrementan la mortalidad natural en la Playa Nancite, está la acción mecánica destructiva y competitiva (por la falta de espacio) que las tortugas realizan durante la anidación. Adicionalmente, el autor principal de este párrafo reportó que en las playas Escobilla y Nancite, la incubación de los huevos toma aproximadamente dos meses (Hughes \& Richard, 1974; García-Romero et al. 2006; Seminoff et al. 2008); mientras que la temporada de arribada se desarrolla en ciclos mensuales (Fig. 5). Esta diferencia temporal intensifica la destrucción masiva de los huevos previos y no incubados, así como también la de crías recién eclosionadas.

\section{AGRADECIMIENTOS}

Agradecemos al Centro Mexicano de la Tortuga con sede en Mazunte, Oaxaca, México, por proveer la base de datos analizada en este trabajo. A la Universidad del Mar, Puerto Ángel, por los recursos técnicos para los análisis. Agradecimientos sinceros para Martha Harfush, Cuauhtémoc Peñaflores y Ernesto Albavera.

\section{BIBLIOGRAFÍA}

Benabib, M. \& Wilson, L. E. (1981). Las tortugas marinas en México. Naturale$z a, 12(3), 157-166$.

Berry, R. J. (1967). Dynamic of the Tortugas (Florida) pink shrimp population. Unpublished Ph.D. Thesis, University of Rhode Island, EE.UU.

Beverton, H. \& Holt, S. (1957). On the dynamics of exploited fish population. New York, EE.UU.: Chapman \& Hall.

Caillouet, C. W. Jr., Duronslet, M. J., Landry, A. M., Revera, D. B., Shaver, D. J., Stanley, K. M., Heinly, R. W. \& Stabenau, E. K. (1991). Sea turtle strandings and shrimp fishing effort in the northwestern Gulf of Mexico, 198689. Fish. Bull., 89(4), 712-718.

Cervantes-Hernández, P., Ramos-Cruz, S. \& Gracia, A. (2006). Evaluación del estado de la pesquería de camarón en el Golfo de Tehuantepec. Hidrobiol., 16(3), 233-239.

Cervantes-Hernández, P., Gallardo-Berumen, M. I., Ramos-Cruz, S., GómezPonce, M. A. \& Gracia, A. (2008a). Análisis de las temporadas de veda en la explotación marina de camarones del Golfo de Tehuantepec, México. Rev. Biol. Mar. Oceanogr., 43(2), $285-$ 294. doi: http://dx.doi.org/10.4067/ S0718-19572008000200007 
Cervantes-Hernández, P., Sánchez-Meraz, B., Serrano-Guzmán, S. J., Frías-Velasco, A., Ramos-Cruz, S. \& Gracia, A. (2008b). Variación interanual de la abundancia de Farfantepenaeus californiensis (Holmes, 1900) en el Golfo de Tehuantepec. Hidrobiol., 18(3), 215-226.

Cervantes-Hernández, P. \& Gracia, A. (2011). Análisis de la mortalidad para el camarón rosado Farfantepenaeus duorarum (Decapoda, Dendrobranchiata) del Banco de Campeche, México. PANAMJAS, 6(2), 100-108.

Cervantes-Hernández, P. (2015). Modelación de las fluctuaciones de la abundancia del camarón café Farfantepenaeus aztecus (Ives, 1891). Tesis Doctoral no publicada. Instituto de Ciencias del Mar y Limnología, Universidad Nacional Autónoma de México.

Cervantes-Hernández, P., Michel-Morfín, E. \& Gallardo-Berumen, M. I. (2016). Reproductive and Recruitment seasons of the Purple Snail Plicopurpura pansa (Gould, 1853) in Oaxaca, Mexico. J. Shellfish Res., 35(4), 99-1005. doi: http://dx.doi. org/10.2983/035.035.0422

Early-Capistrán, M. M. (2014). Lo vulnerable y lo sanguinario: explotación histórica de tortugas y tiburones en la costa de Oaxaca. En R. Macip-Ríos \& O. Espinosa-Santiago (Eds.), Problemas ambientales asociados al desarrollo (pp. 51-69). Puebla, México: Benemérita Universidad Autónoma de Puebla.

Enciso-Saracho, F. (2007). Descripción de anomalías en tortugas golfinas Lepidochelys olivacea, observadas en playa Escobilla, Oaxaca, durante la temporada 1985. En G. Fuentes-Mascorro (Ed.), XXV Aniversario de conservación e investigación en tortuga marina: Tomo II santuario "La Escobilla", un compromiso de conservación con la humanidad (pp. 67-75). Oaxaca, México: Universidad Autónoma Benito Juárez.

Fernández-Álamo, M. A., SanvicenteAñorve, L. \& Alameda-De-La-Mora, G. (2000). Copepod assemblages in the Gulf of Tehuantepec, Mexico. Crustaceana, 73(9), 1139-1153. doi: 10.1163/156854000505137

Frías-Velasco, A. (2004). Regionalización de los golfos de Tehuantepec, $\mathrm{Pa}$ pagayo, Panamá y áreas adyacentes mediante la biomasa fitoplanctónica estimada a partir de imágenes de satélite. Tesis Licenciatura. Universidad del Mar, Puerto Ángel, Oaxaca.

Gallegos-García, A. \& Barberán-Falcón, J. (1998). Surgencia eólica. En M. Tapia-García (Ed.), El Golfo de Tehuantepec: el ecosistema y sus recursos (pp. 27-34). CDMX, México: Universidad Autónoma Metropolitana-Iztapalapa.

García-Romero, M. E., Hernández-Dueñas, L. M., García-Castañeda, B., Santos-Soto, A. \& Meyer-Willerer, A. O. (2006). Protección y conservación de la tortuga marina de la zona costera de Michoacán, México. A. I. A., 11(2), 15-21.

Haddon, M. (2011). Modelling and quantitative methods in fisheries. London, England: Chapman \& Hall CRC Press. Hirth, H. F. \& Schafter, W. (1974). Survival rates of the green turtle, Chelonia mydas, necessary to maintain stable populations. Copeia, 2, 544-546.

Hood, G. M. (2006). PopTools version 2.7.5. Albany, Western Australia: CSIRO.

Hughes, D. A. \& Richard, J. D. (1974). The Nesting of the Pacific Ridley Turtle Lepidochelys olivacea on Playa Nancite, Costa Rica. Mar. Biol., 24, 97-107.

INAPESCA. Instituto Nacional de Pesca. (2007a). Resultado del análisis de 
poblaciones de camarón del litoral del Pacifico para implementar la veda durante el 2007. México: Secretaría de Medio Ambiente, Recursos Naturales y Pesca, México.

INAPESCA. Instituto Nacional de Pesca. (2007b). Resultados de muestreos de las poblaciones de camarón, durante la veda del 2007 litoral del Pacífico. México: Secretaría de Medio Ambiente, Recursos Naturales y Pesca, México. Lara-Lara, J. R., Robles-Jerero, E. G., Bazán-Guzmán, M. C. \& MillánNúñez, E. (1998). Productividad del fitoplancton. En M. Tapia-García (Ed.), El Golfo de Tehuantepec: el ecosistema y sus recursos (pp. 5158). México: Universidad Autónoma Metropolitana-Iztapalapa.

Makridakis, S., Wheelwright, S. \& McGee, V. (1983). Forecasting: methods and applications. New York, EE.UU.: Wiley.

Margaleff, M. (1978). Radiación y energía en ecología. Barcelona, España: OMEGA.

Márquez-M., R., Villanueva-O., A. \& Peñaflores-S., C. (1976). Sinopsis de datos biológicos sobre la tortuga golfina Lepidochelys olivacea (Eschscholtz 1829). CDMX, México: SIC-SP-INP.

Márquez-M., R., Villanueva-O., A., Peñaflores-S., C. \& Ríos-O., D. (1982). Situación actual y recomendaciones para el manejo de las tortugas de la costa occidental mexicana, en especial la tortuga golfina Lepidochelys olivacea. Ciencia Pesquera, 3, 83-91.

Márquez-M., R. (1996). Las tortugas marinas y nuestro tiempo. CDMX, México: Fondo de Cultura Económica.

Márquez-M., R. (2014). México y las tortugas marinas. En R. MárquezMillán \& M. Garduño-Dionate (Eds.),
Tortugas marinas (pp. 9-12). CDMX, México: Instituto Nacional de la Pesca. Márquez-M., R., Jiménez-Quiroz, M. C., Peñaflores-S., C. \& Díaz-Flores, J. (2014). México y las tortugas marinas. En R. Márquez-Millán \& M. GarduñoDionate (Eds.), Tortugas marinas (pp. 13-47). CDMX, México: Instituto Nacional de la Pesca.

Meraz-Hernando, J. F. \& Ávila-Barrientos, J. (2007). Efecto de la selección natural sobre la eclosión de los huevos de tortuga golfina (Lepidochelys olivacea). En G. Fuentes-Mascorro (Ed.), $X X V$ Aniversario de conservación e investigación en tortuga marina: Tomo II santuario "La Escobilla", un compromiso de conservación con la humanidad (pp. 60-65). Oaxaca, México: Universidad Autónoma Benito Juárez.

Peñaflores-Salazar, C., Vasconcelos, J., Albavera, E. \& Jiménez-Quiroz, M. C. (2001). Especies sujetas a protección especial. Tortuga golfina. En M. A. Cisneros-Mata, L. Meléndez-Moreno, E. Zárate-Becerra, M. T. GasparDillanes, L. C. López-González, C. Saucedo-Ruiz \& J. Tovar-Ávila (Eds.), Sustentabilidad y pesca responsable en México, evaluación y manejo (pp. 1001-1021). CDMX, México: Instituto Nacional de la Pesca.

PESCA. Secretaría de Pesca. (1980-1989). Anuario estadístico de pesca 1980. México: Departamento de Pesca.

PESCA. Secretaría de Pesca. (1995-2007). Anuario estadístico de pesca 1995. Tlalpan, México: Secretaría de Medio Ambiente, Recursos Naturales y Pesca.

Ramos-Cruz, S., Sánchez-Meraz, B., Carrasco-Ayuso, F. \& Cervantes-Hernández, P. (2006). Estimación de la tasa de mortalidad natural de Farfantepenaeus californiensis (Holmes, 1900) y Litopenaeus 
vannamei (Boone, 1931), en la zona costera del Golfo de Tehuantepec, México. Rev. Biol. Mar. Oceanog., 41(2), 221-229. doi: http://dx.doi.org/10.4067/ S0718-19572006000200010

Ricker, W. E. (1975). Computation and interpretation of biological statistics of fish populations. Bull. Fish. Res. Board. Can., 191, 1-382.

SAGARPA. Secretaría de Agricultura, Ganadería, Desarrollo Rural, Pesca y Alimentación. (2007). Norma Oficial Mexicana NOM-061-PESC-2006. (Publicado en DOF: 22/01/2007). México: Diario Oficial de la Federación.

SEMARNAP. Secretaría de Medio Ambiente y Recursos Naturales. (19961996). Norma Oficial Mexicana NOMEM-001-1996 y EM-002-PESC-1996. (Publicado en DOF: 18/03/1996). México: Diario Oficial de la Federación.

Seminoff, J. A., Reséndiz-Hidalgo, A., Jiménez de Reséndiz, B., Wallace, J. N. \& Todd-Jones, T. (2008). Tortugas marinas. En G. D. Danemann \& E. Ezcurra
(Eds.), Bahía de los Ángeles: recursos naturales y comunidad (pp. 457-493). CDMX, México: Secretaría de Medio Ambiente y Recursos Naturales.

StatSoft, Inc. (2002). STATISTICA 7.0 for Windows. Tulsa, Oklahoma, EE.UU.: StatSoft. Tapia-García, M. \& Gutiérrez-Díaz, B. (1998). Recursos pesqueros de los estados de Oaxaca y Chiapas. En M. Tapia-García (Ed.), El Golfo de Tehuantepec: el ecosistema y sus recursos (pp. 1249-1262). CDMX, México: Universidad Autónoma Metropolitana. Trinidad, H. \& Wilson, J. (2000). The bioeconomics of sea turtle conservation and use in Mexico: History of exploitation and conservation policies for the olive ridley (Lepidochelys olivacea). Corva1lis, EE.UU.: International Institute of Fisheries Economics and Trade (IIFET).

Vetter, E. F. (1988). Estimation of natural mortality in fish stocks: a review. Fish. Bull., 86(1), 25-43.

Zar, H. J. (1999). Biostatistical analysis. New Jersey, EE.UU.: Prentice Hall. 